\title{
Controlled-NOT gate operating with single photons
}

M. A. Pooley, ${ }^{1,2, \text { a) }}{ }^{2}$ D. J. P. Ellis, ${ }^{1}$ R. B. Patel, ${ }^{1,2}$ A. J. Bennett, ${ }^{1}$ K. H. A. Chan, ${ }^{1,2}$

I. Farrer, ${ }^{2}$ D. A. Ritchie, ${ }^{2}$ and A. J. Shields ${ }^{1}$

1) Toshiba Research Europe Limited, Cambridge Research Laboratory, 208 Science Park, Milton Road, Cambridge, CB4 OGZ, U.K.

${ }^{2)}$ Cavendish Laboratory, Cambridge University, J. J. Thomson Avenue, Cambridge, CB3 OHE, U.K.

The initial proposal for scalable optical quantum computing required single photon sources, linear optical elements such as beamsplitters and phaseshifters, and photon detection. Here we demonstrate a two qubit gate using indistinguishable photons from a quantum dot in a pillar microcavity. As the emitter, the optical circuitry, and the detectors are all semiconductor, this is a promising approach towards creating a fully integrated device for scalable quantum computing.

a) Electronic mail: map57@cam.ac.uk 
Quantum computing offers a drastic increase in the speed of solving parallelizable computational tasks. Optical quantum computing, where photons are used as quantum bits (qubits), has many advantages. Photons have long coherence times and travel fast, allowing connection between remote nodes of a quantum computer, and they can easily be manipulated at the single qubit level. A promising approach to optical quantum computing, linear optics quantum computing (LOQC $)^{1}$, requires triggered single photon sources which supply photons into optical modes in well-defined states. Several protocols have been proposed that reduce the resource overhead required to build an optical quantum computer ${ }^{2-4}$, but the ability to initialize a certain photon state is still of central importance. Significant progress in experimental work on few-qubit gates has been made using probabilistic sources, such as optically pumped parametric down-conversion crystals $^{5-8}$. However, the Poissonian statistics which govern such sources can seriously impair the performance of quantum logic gates, precluding systems containing many gates.

This has motivated much research into on-demand single photon emission. A variety of triggered single photon sources have been demonstrated including, molecules ${ }^{9,10}$, trapped ions ${ }^{11}$, color centers, and quantum dots $(\mathrm{QDs})^{12}$. Semiconductor sources are particularly promising as many emitters can be fabricated on a small area and they can be integrated directly into semiconductor waveguide technology. Here we present a two qubit gate in which a single QD is used as the photon source and the optical circuitry is realized using a semiconductor waveguide. The Controlled-NOT (CNOT) gate we demonstrate is the basic building block of quantum logic, since in combination with one qubit gates it can be used to perform any quantum operation.

In our work, the photon source consists of a single self-assembled InAs QD embedded inside a $1.5 \mu \mathrm{m}$ diameter pillar microcavity with a quality factor, $Q \sim 9000$. The QD exciton transition emits at a wavelength of $\lambda=931 \mathrm{~nm}$, which is resonant with the $\mathrm{HE}_{11}$ cavity mode of the pillar. The planar structure of the pillar microcavity, which is grown by molecular beam epitaxy, consists of 17 (25) GaAs/AlGaAs distributed Bragg reflector periods above (below) a one-wavelength thick GaAs cavity centered on a single layer of selfassembled InAs QDs. The pillar is defined using reactive ion etching, see Figures 1a - b. The advantage of using a pillar microcavity structure is two-fold, (1) Purcell enhancement ${ }^{13}$ reduces the radiative lifetime relative to the coherence time, increasing the probability that consecutive photons are indistinguishable; (2) Collection efficiency is significantly increased 

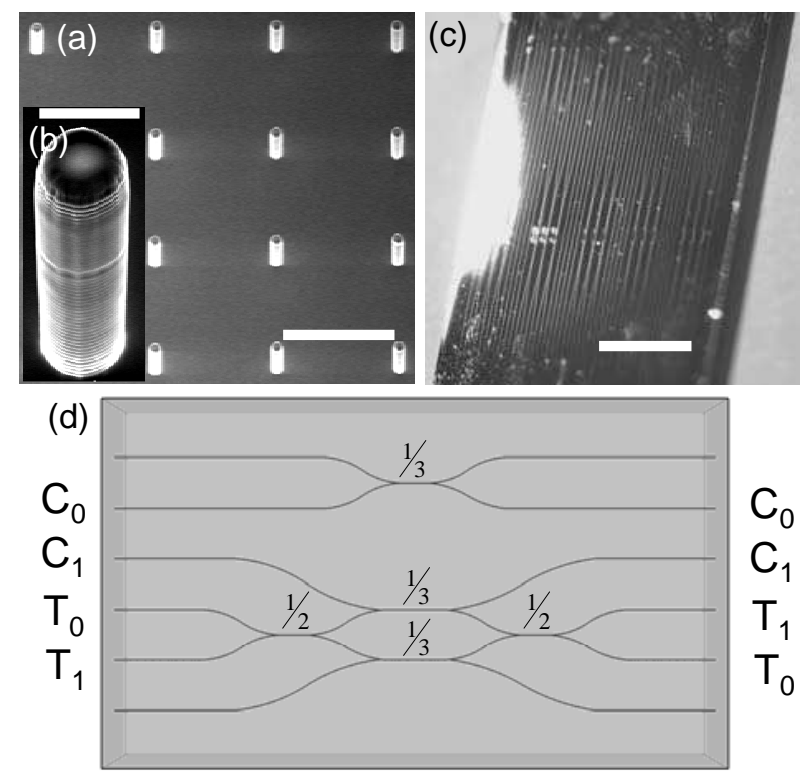

FIG. 1. (Color online) SEM images of (a) an array of pillar microcavities, and (b) a single pillar microcavity (scale bars $20 \mu \mathrm{m}$ and $2 \mu \mathrm{m}$ respectively). (c) a semiconductor waveguide chip (scale bar $5 \mathrm{~mm}$ ) (d) Schematic diagram of a single waveguide circuit.

as QD emission is coupled into the cavity mode.

The pillar is held at a temperature of $4.5 \mathrm{~K}$ and the QD is excited quasi-resonantly one LOphonon $(\sim 32 \mathrm{meV})$ above the exciton energy level by a pulsed 1364 meV modelocked laser with a repetition frequency of $80 \mathrm{MHz}$. Quasi-resonant excitation prevents excess carrier generation in the wetting layer, thus reducing the effective radiative lifetime and increasing the coherence time of the resulting excitons. In addition, excitation through a phonon resonance allows selective excitation of only one polarization state, as the polarization of the laser photon is transferred to the spin of the exciton. This doubles the internal quantum efficiency of the source, relative to quasi-resonant excitation schemes that promote the system into an excited state which can decay into either of the exciton polarization states. Exciting the source under these conditions, at a small fraction of saturation, we measure a radiative lifetime of $\tau_{R}=106 \mathrm{ps}$, a coherence time of $\tau_{C}=148 \mathrm{ps}$, and the photon emission rate is $\sim 5 \mathrm{MHz}$ into an $\mathrm{NA}=0.42$ microscope objective above the emitter.

The CNOT input state requires two photons, both of which are emitted from the same QD. An unbalanced Michelson interferometer is used to split each laser pulse into two pulses separated by $1.95 \mathrm{~ns}$, thus allowing the QD to be excited twice in quick succession each laser 


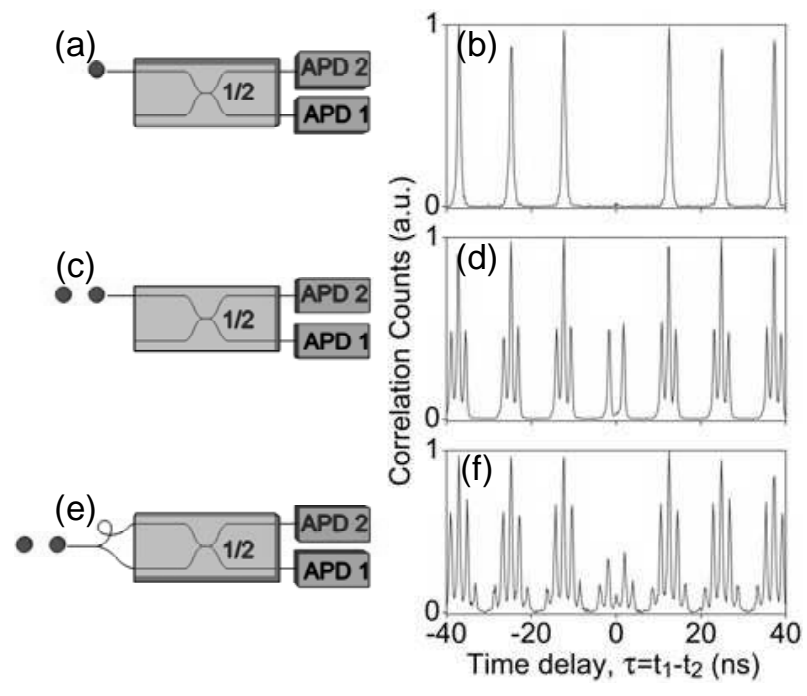

FIG. 2. (Color online) (a,b), Pulsed second-order autocorrelation measurement yields $g^{(2)}(0)=$ $0.013 \pm 0.004$. (c,d), Pulsed autocorrelation measurement when the QD is excited twice in quick succession shows that the probability of either excitation resulting in multi-photon emission to be $g=0.0063 \pm 0.0005$. (e,f), Pulsed two photon interference measurement using a $\frac{1}{2}$ coupler yields a two photon interference visibility of $V^{(2)}=0.72 \pm 0.05$.

repetition cycle, as required for our state preparation scheme.

The single photon nature of the source is confirmed by a pulsed second-order autocorrelation measurement in a waveguide circuit shown in Figure 2a. The results, seen in Figure $2 \mathrm{~b}$, show that the multi-photon emission rate is just $(1.3 \pm 0.4) \%$ that of a Possonian emitter of the same intensity. Also shown in Figure $2 \mathrm{~d}$ is a second-order autocorrelation measurement when the QD is excited twice per cycle, under the conditions needed to implement the CNOT operation. From this measurement it is possible to extract the probability that either of the emission events are multi-photon, $g$. The residual between the data and a double Lorentzian fit to the peaks at \pm 1.95 ns yields a probability of multi-photon emission in either pulse of $g=0.0063 \pm 0.0005$.

Each pair of photons must be indistinguishable in order to maximize the degree of two photon interference, $V^{(2)}$, which determines the probability of the two qubits interacting correctly when the input state has $|1\rangle_{C}$. A two photon interference measurement ${ }^{14}$, shown in Figure 2f, using an isolated $\frac{1}{2}$ directional coupler, yields $V^{(2)}=0.72 \pm 0.05$. This is comparable with the highest reported for single photon semiconductor sources ${ }^{15,16}$.

The optical circuit is realized using a semiconductor waveguide, shown in Figure 1c, in 
which directional couplers are used to perform the function of beamsplitters. The circuit implements the CNOT gate proposed by Ralph et al. ${ }^{17}$ and consists of a network of directional couplers with coupling ratios of $\frac{1}{2}$ and $\frac{1}{3}$ as shown schematically in Figure 1d. Each waveguide core has cross-sectional dimensions of $3.8 \times 3.8 \mu \mathrm{m}$, and the complete CNOT circuit has lateral dimensions of $1.25 \times 32.5 \mathrm{~mm}$. The semiconductor waveguide is fabricated in silica on a silicon substrate using standard semiconductor processing. Photons are confined within the waveguides by the refractive index contrast between the core and cladding regions. The coupling ratio of a directional coupler is wavelength dependent and can be precisely tuned by adjusting the interaction length. Isolated test couplers, nominally identical to those in the network, were formed adjacent to the circuit to permit the direct measurement of the actual coupling ratios inside the CNOT circuit. The couplers used in this work have coupling ratios of $R_{1 / 3}=0.345$ and $R_{1 / 2}=0.495$.

The two qubits are path encoded, considering the logical basis where each qubit has a single photon in one path and zero photons in the other, the input states are $|C\rangle|T\rangle=|C T\rangle=$ $|00\rangle_{C T},|01\rangle_{C T},|10\rangle_{C T},|11\rangle_{C T}$. The QD emission is coupled into a 50/50 fiber beamsplitter, with one output connected to a target path input and the other output connected to a control path input via a 1.95 ns delay. If the first photon takes the control path and the second takes the target path the two photons enter the circuit simultaneously and the desired input state is realized. Light is coupled into and out of the waveguide using fiber arrays which are butt-coupled with index matching fluid to the waveguide. Polarization-maintaining (PM) single-mode fiber is used for the inputs and multi-mode fiber is used to collect from the outputs. Optical loss in the circuit is negligible, but typically the insertion loss coupling from PM fibre to a waveguide is $50 \%$.

For each input state, the QD emission is coupled into the corresponding waveguide inputs and four time-resolved correlation measurements are simultaneously acquired between control and target output paths, one for each of the possible output states. Each set of correlation measurements requires 30 minutes accumulation time. The coincidence counts at zero delay $(\tau=0)$ correspond to cases where the correct input state is realized, the area of these peaks are used to construct the truth table.

Figure 3 shows the experimental correlation measurements (filled red bars), along with predicted correlation curves (solid black lines). The predicted curves are calculated by considering the possible paths that the photons can take through the waveguide network, 


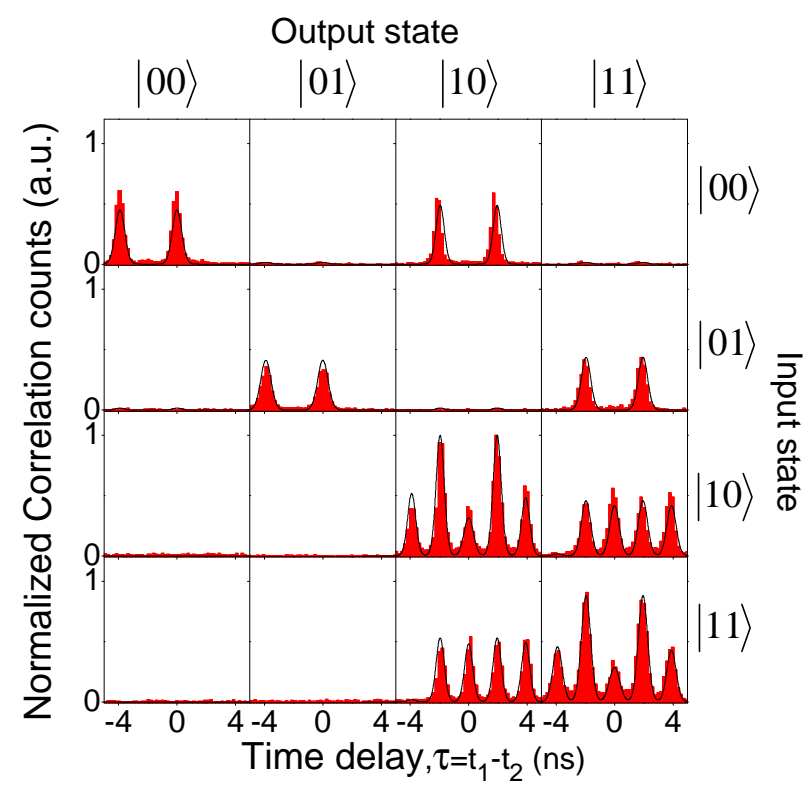

FIG. 3. (Color online) Each pane corresponds to a correlation measurement for a single input and output combination. Measured data are shown as filled red bars. Solid black lines show calculated curves for the expected correlations given the measured coupler ratios, single photon interference visibility, and two photon interference visibility. The peaks at $\tau=0$ correspond to events where both photons entered the circuit simultaneously, and the area of these peaks is used to extract the truth table for the operation.

with the probability of each path adjusted to account for the experimentally determined coupler reflectivities and single-photon interference visibility. For the $\langle 10 \mid 10\rangle$ and $\langle 11 \mid 11\rangle$ correlations the central $\tau=0$ peaks are dependent on two-photon interference effects, these peaks are calculated using a wavepacket approach similar to Legero et al..$^{18,19}$, in which the temporal properties of the photons $\left(\tau_{R}\right.$ and $\left.\tau_{C}\right)$ and the waveguide imperfections are considered.

From the $\langle 00 \mid 00\rangle$ and $\langle 00 \mid 01\rangle$ correlations it is possible to extract a single photon interference visibility of $V^{(1)}=0.97$, which gives the probability of a single photon traversing the Mach-Zehnder interferometer correctly in the absence of any qubit-qubit interaction. The two photon interference visibility, which can be obtained from the $\langle 10 \mid 10\rangle$ and $\langle 11 \mid 11\rangle$ correlations, is limited not only by the distinguishability of the photons but also by properties of waveguide circuit, specifically the single photon interference visibility and the non-optimal coupler ratios. The data agrees well with the predicted correlation curves which are calcu- 

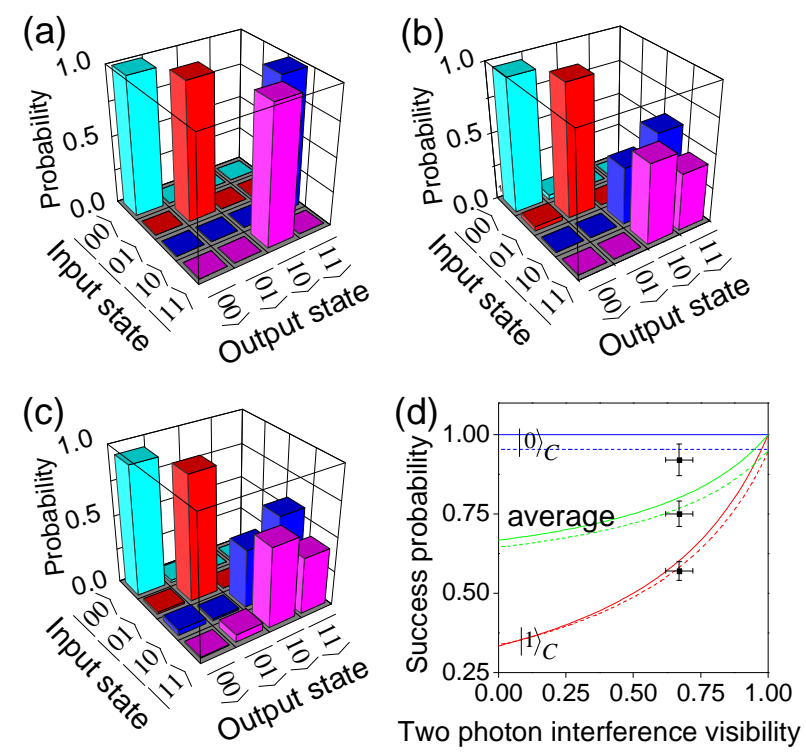

FIG. 4. (Color online) (a) Truth table for an ideal CNOT gate. (b) Predicted truth table for the circuit and photon source used in this experiment based on the experimentally achieved two photon interference visibility, single photon interference visibility, and coupler ratios. (c) Experimentally achieved truth table. (d) Success probability as a function of two photon interference visibility for the $|0\rangle_{C}$ input states (blue), $|1\rangle_{C}$ input states (red) and averaged over all input states (green). Solid lines are for an ideal circuit, dashed lines are for the circuit realized experimentally. The values extracted from the measured truth table are shown as black squares, error bars are Poissonian errors in the number of counts.

lated using the measured values of $V^{(1)}, R_{1 / 3}, R_{1 / 2}$ and $V^{(2)}=0.67$, which is consistent with the two photon visibility obtained for an isolated $\frac{1}{2}$ coupler.

Figures 4a-c show the measured truth table, along with those for ideal CNOT gate operation, and operation with photon interference visibilities of $V^{(1)}=0.97$ and $V^{(2)}=0.67$. Each element of the truth tables in figure 4 is extracted from the area of the coincidence $(\tau=0)$ peak in the corresponding correlation measurement of figure 3. Each truth table row corresponds to a single input state, and is normalized by the total area of the four coincidence peaks in the associated outputs. The success probability for an input state is then given by the value of the truth table element which corresponds to the correct output state.

The success probability as a function of $V^{(2)}$, for an ideal CNOT circuit (solid lines) and the circuit realized experimentally in this work (dashed lines), is shown in Figure 4d. The 
success probabilities obtained experimentally are shown as filled black squares in the same figure. There is good agreement between the measured and calculated values. The truth table for the operation is extracted from the coincidence measurements by integrating all counts in a window of $1.95 \mathrm{~ns}$ centered at $\tau=0$. The probability of the gate succeeding averaged over all inputs is $71 \%$. Taking a window of $0.6 \mathrm{~ns}$ increases the overall success probability to $75 \%$, while preserving $60 \%$ of the coincidences. In principle, detectors with a faster response time could further improve the measured success rate. It is clear that increasing the indistinguishability between photons is the key to improving the success probability of the gate.

In our experiment the degree of photon indistinguishability is determined by the ratio of the radiative decay time and coherence time of the exciton within the QD. For the quasi-resonant excitation scheme we use, residual decoherence is caused by interaction of the exciton with the solid-state environment. There is potential to increase the coherence time through the use of coherent Raman excitation schemes ${ }^{20,21}$. Even better, resonance fluorescence measurements ${ }^{22}$ can transfer the coherence properties of the excitation laser onto the exciton photon, significantly lengthening the coherence times toward that of the driving laser. Ideally, this excitation scheme could be used to drive multiple quantum dots to generate identical photons. The natural inhomogeneity in linewidth for each dot would be suppressed if they were driven with the same laser, and the inhomogeneity in emission energy could be suppressed with electric field tuning of the transitions, as has recently been demonstrated ${ }^{19}$.

In conclusion, we have demonstrated logical operation of an all semiconductor optical CNOT gate operating with indistinguishable single photons. These results are promising for the scalability of future photonic quantum computing technology. Extending our scheme to incorporate multiple single photon sources will allow deterministic preparation of multiphoton input states. Integration of the photon source and detectors directly into photonic circuitry will allow further miniaturization and reduction of the resources required.

\section{REFERENCES}

${ }^{1}$ E. Knill, R. Laflamme, and G. J. Milburn, Nature, vol. 409, pp. 46-52, Jan. 2001.

${ }^{2}$ R. Raussendorf and H. J. Briegel, Phys. Rev. Lett., vol. 86, pp. 5188-5191, May 2001. 
${ }^{3}$ D. E. Browne and T. Rudolph, Phys. Rev. Lett., vol. 95, pp. 010501-, June 2005.

${ }^{4}$ P. Kok, W. J. Munro, K. Nemoto, T. C. Ralph, J. P. Dowling, and G. J. Milburn, Rev. Mod. Phys., vol. 79, pp. 135-174, Jan. 2007.

${ }^{5}$ S. Gasparoni, J.-W. Pan, P. Walther, T. Rudolph, and A. Zeilinger, Phys. Rev. Lett., vol. 93, pp. 020504-, July 2004.

${ }^{6}$ J. L. O’Brien, G. J. Pryde, A. G. White, T. C. Ralph, and D. Branning, Nature, vol. 426, pp. 264-267, Nov. 2003.

${ }^{7}$ R. Okamoto, H. F. Hofmann, S. Takeuchi, and K. Sasaki, Phys. Rev. Lett., vol. 95, pp. 210506-, Nov. 2005.

${ }^{8}$ A. Politi, M. J. Cryan, J. G. Rarity, S. Yu, and J. L. O’Brien, Science, vol. 320, no. 5876, pp. 646-649, 2008.

${ }^{9}$ B. Lounis and W. E. Moerner, Nature, vol. 407, pp. 491-493, Sept. 2000.

${ }^{10}$ C. Brunel, B. Lounis, P. Tamarat, and M. Orrit, Phys. Rev. Lett., vol. 83, pp. 2722-2725, Oct. 1999.

${ }^{11}$ A. Kuhn, M. Hennrich, and G. Rempe, Phys. Rev. Lett., vol. 89, pp. 067901-, July 2002.

${ }^{12}$ P. Michler, A. Kiraz, C. Becher, W. V. Schoenfeld, Lidong Zhang, E. Hu, and A. Imamoglu Science, vol. 290, pp. 2282-2285, Dec. 2000.

${ }^{13}$ E. M. Purcell, Phys. Rev., vol. 69, p. 681, 1946.

${ }^{14}$ C. K. Hong, Z. Y. Ou, and L. Mandel, Phys. Rev. Lett., vol. 59, pp. 2044-2046, Nov. 1987.

${ }^{15}$ C. Santori, D. Fattal, J. Vuckovic, G. S. Solomon, and Y. Yamamoto, Nature, vol. 419, pp. 594-597, Oct. 2002.

${ }^{16}$ A. J. Bennett, R. B. Patel, A. J. Shields, K. Cooper, P. Atkinson, C. A. Nicoll, and D. A. Ritchie, Appl. Phys. Lett., vol. 92, pp. 193503-3, May 2008.

${ }^{17}$ T. C. Ralph, N. K. Langford, T. B. Bell, and A. G. White, Phys. Rev. A, vol. 65, pp. 062324-, June 2002.

${ }^{18}$ T. Legero, T. Wilk, A. Kuhn, and G. Rempe, Appl. Phys. B, vol. 77, pp. 979-802, 2004.

${ }^{19}$ R. B. Patel, A. J. Bennett, I. Farrer, C. A. Nicoll, D. A. Ritchie, and A. J. Shields, Nat Photon, vol. 4, . 632-635, 2010.

${ }^{20}$ C. Santori, D. Fattal, K.-M. C. Fu, P. E. Barclay, and R. G. Beausoleil, New Journal of Physics, vol. 11, no. 12, pp. 123009-, 2009.

${ }^{21}$ A. Kiraz, M. Atature, and A. Imamoglu, Phys. Rev. A, vol. 69, pp. 032305-, Mar. 2004.

${ }^{22}$ H. S. Nguyen, G. Sallen, C. Voisin, Ph. Roussignol, C. Diederichs, and G. Cassabois, Appl. 
Phys. Lett., vol. 99, pp. 261904, Dec. 2011. 